\title{
interview
}

\section{A happy catastrophe}

\section{The application of catastrophe theory to high-harmonic generation is creating opportunities for optimizing the spectral intensity of extreme-ultraviolet light and characterizing attosecond pulses, explains Oren Raz from the Weizmann Institute of Science in Israel.}

\begin{abstract}
What is catastrophe theory, and what is it used for?

Catastrophe theory is a universal way of looking at singularities. It is a beautiful and powerful mathematical tool that is used throughout many areas of physics and mathematics. Pioneered by mathematicians René Thom, Vladimir Arnold and Christopher Zeeman in the 1960s and 1970s, catastrophe theory provides a set of analytic mathematical functions known as 'catastrophes' that can be used to describe what happens near singularities in a system. The specific name and shape of a catastrophe depends on the number of control paameters within the system. The simplest catastrophes are folds or cusps, while more complex ones are known as swallow tails or butterflies, due to their shape. In the field of optics, scientists have known for a long time that catastrophe theory can be used to describe the light patterns generated by the reflection and refraction of light rays from curved surfaces such as a wine glass or water in a swimming pool, for example. In these cases, the bright lines of enhanced intensity are known as optical caustics, with focal points and lines acting as singularities.
\end{abstract}

Where did you get the idea of applying catastrophe theory to high-harmonic generation and attosecond science? Interestingly, the original idea for this technique came from a completely different field - the study of Airy beams. During that work, we came across a series of old papers written by Michael Berry, a theoretical physicist at the University of Bristol in the UK. We discovered that many of his papers made use of catastrophe theory, which was new and unfamiliar to us at the time (the Airy beam is itself a caustic). We then saw that similar ideas could be directly applied to our work with attosecond light pulses; we realized that catastrophe theory could be used not only to explain Airy beams, but also to study the process of high-harmonic generation and predict locations of enhanced intensity within spectra, which we call spectral caustics.

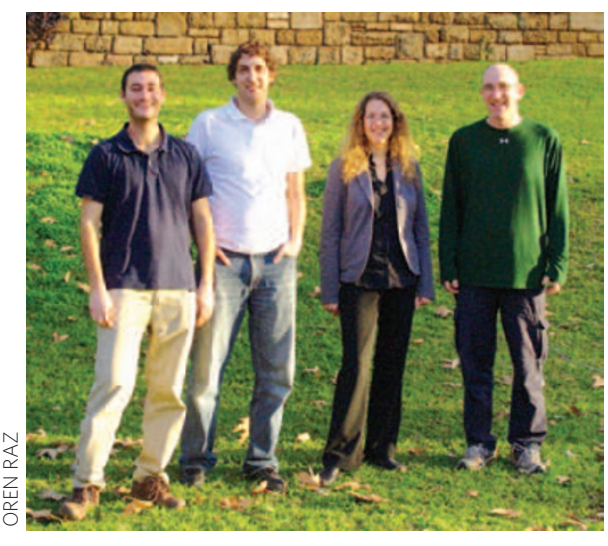

Left to right: Oren Pedatzur, Barry Brunner, Nirit Dudovich and Oren Raz. The researchers, from the Weizmann Institute of Science in Israel, have successfully applied catastrophe theory and caustics to the spectra of attosecond pulses in the extreme ultraviolet to find regions of enhanced intensity.

Were you surprised by your results? After learning about caustics and catastrophe theory, what we saw came as no surprise. We also found that caustics also existed in spectral high-harmonic data we had already collected. Beyond the swallow tail caustic of our current work, we also know about the predicted existence of a few other caustics relating to higher-parameter spaces that we haven't yet measured.

\section{"We hope to use caustics in the characterization of attosecond pulses, which would represent a powerful tool for attosecond metrology."}

What are the implications and consequences of your work?

Our work offers both experimental and theoretical opportunities. Attosecond pulses are naturally very short and have a very wide spectrum. In many applications, however, you don't want or need such a wide spectrum; rather you filter the pulses to obtain a coherent source for a particular wavelength in the extremeultraviolet region. The ideal solution would be a spectral lens capable of focusing the intensity from a broad range of frequencies into a single frequency. Unfortunately researchers have yet to develop such a technique. A caustic can be thought of as an alternative to a spectral lens because it provides local enhancement in a certain part of the spectrum. One possibility is using catastrophe theory to determine how to create a caustic in the spectral region of interest. There are also a number of other experimental possibilities. Researchers could use caustics to label molecular levels in spectroscopic analysis, for example. Catastrophe theory is interesting from a theoretical point of view for high-harmonic generation because it is complementary to both the classical three-step model, which is simple to use but has limitations, and quantum-mechanical calculations, which are accurate but not very intuitive.

\section{What are your plans for future work in} this area?

We are thinking of trying two things. First, the lack of lenses in the extreme-ultraviolet region has always been a great limitation for the field. We would therefore like to investigate the idea of using caustics to provide both spectral and spatial focusing. Bringing together spectral and spatial caustics would in principle allow a specific harmonic to be focused at a specific location. This would be an important breakthrough, and it is something we are currently working on. The second area is the temporal characterization of pulses in attosecond science. At present, scientists can easily measure the spectrum of attosecond pulses, but cannot easily measure their phase. Catastrophe theory can be used to determine the exact functional form and the phase around these caustics. We hope to use caustics in the characterization of attosecond pulses, which would represent a powerful tool for attosecond metrology.

\section{INTERVIEW BY OLIVER GRAYDON}

Oren Raz and his co-workers have a Letter on spectral caustics and high-harmonic generation on page 170 of this issue. 